$$
\text { نمذجة خلايا الخزن النانو بلورية }
$$

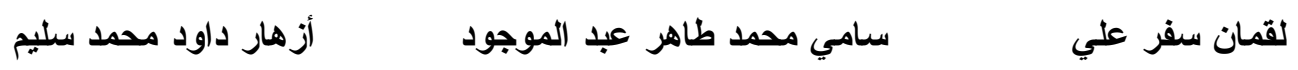

$$
\text { جامعة الموصل / كلية الهندسة / قسم الهندسة الكهربائية }
$$

\title{
الخلاصة
}

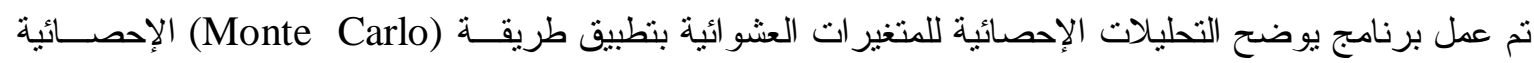

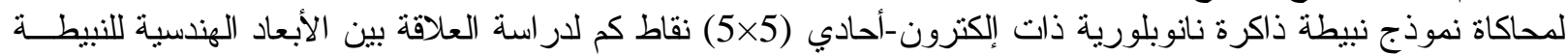

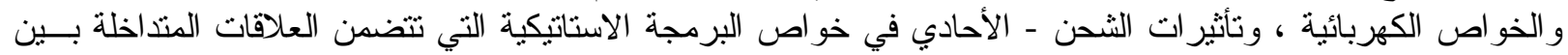

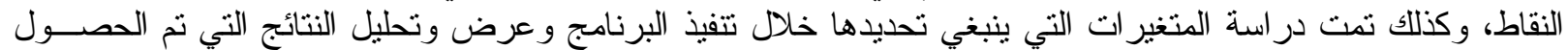

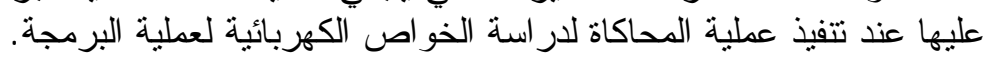

\section{Modeling of Nanocrystal Storage Cells}

\author{
L. S. Ali \\ S. M. T. Abdul Mawjoud \\ A. D. Mohammed Saleem
}

University of Mosul - College of Engineering- Electrical Engineering Department

\begin{abstract}
The computer program is prepared for applying Montecarlo simulation and modeling for single-electron nanocrystal memories. The nanocrystal memory device of $(5 \times 5)$ quantum dots is used for studying the relationship between, geometrical dimensions, electrical characteristics and charging effects for single electron static programming characteristics. The nanocrystal inter-dot effects are included. All parameters got in the memory simulation programming are studied and discussed.
\end{abstract}

Keywords: Nanocrystal Memories. 
: 1

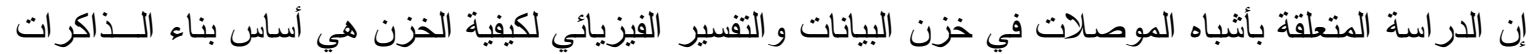

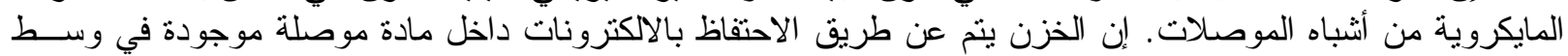
عازل ( بوابة عائمة ) م المباه قامت مجموعة من الباحثن الكوريين [1] (Yu et al., 2001) باقتر اح نموذج خلية ذاكرة الكتــرون - أحسادي جديدة. وقد أظهرت نتائج التمثيل طريقة عمل مقبولة لخين لخلية الذاكرة.

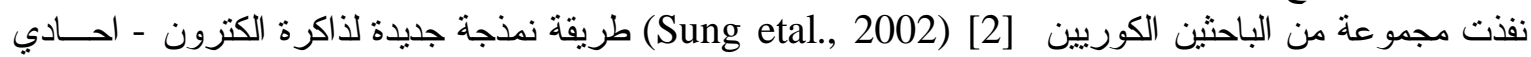

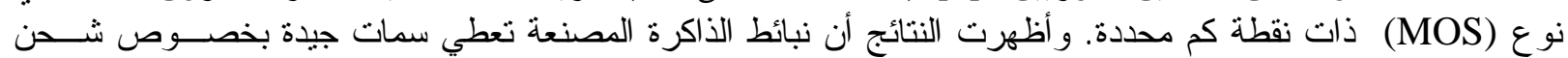

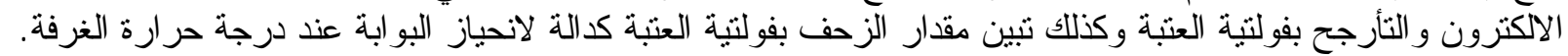

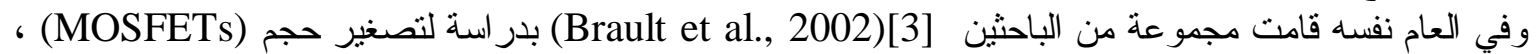

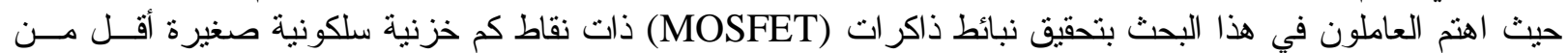

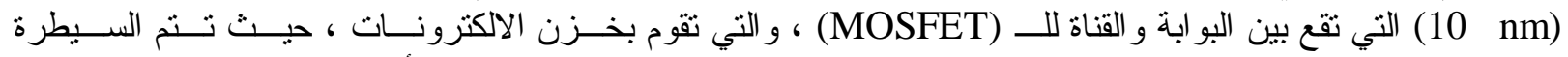

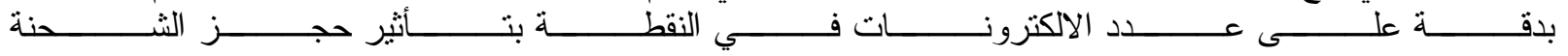
. (Coulomb Blocked) (CB)

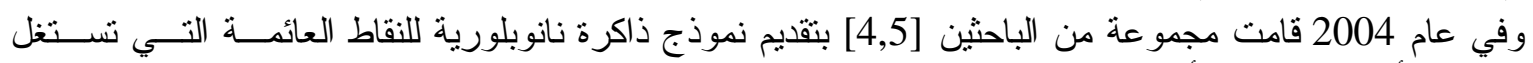

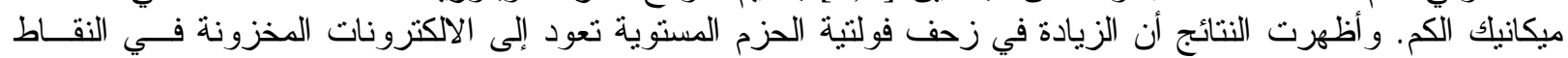

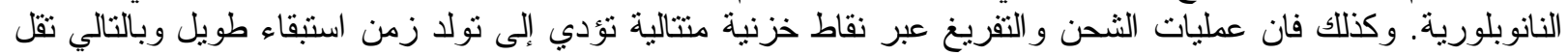

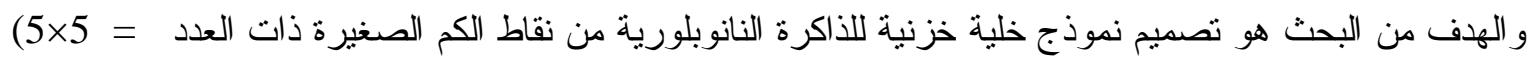

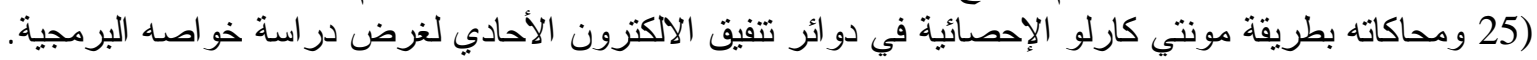

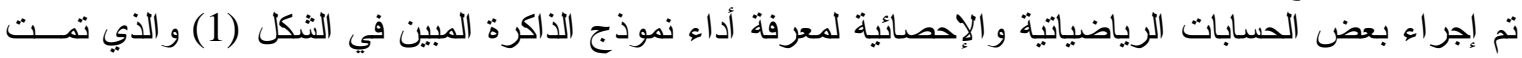

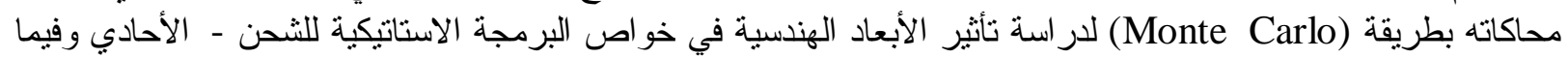
يأتي تفاصيل ذلك : تم افتر اض درجة الحر ارة (T) تساوي (300K) و إن زمن فترة البرمجة طويلة بما يكفي للوصول لحالـــة حجـز

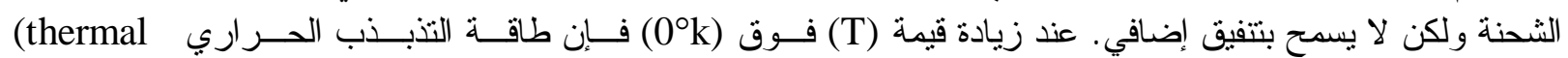

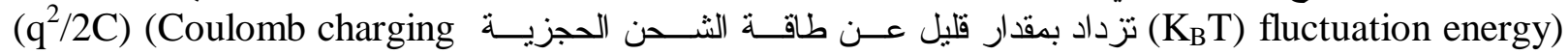

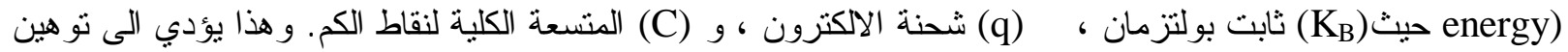

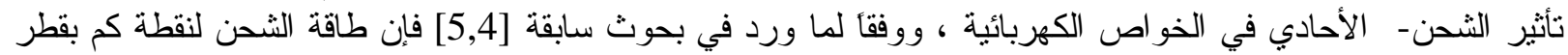

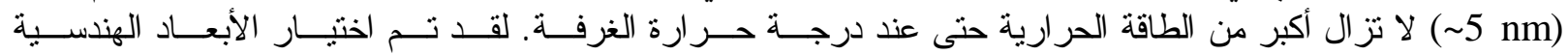

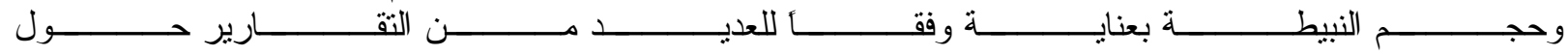

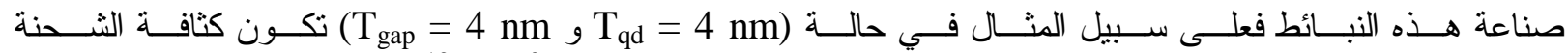

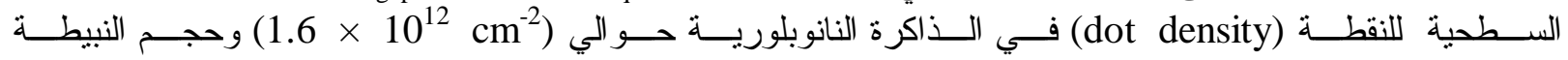
(L/W = 20 nm / 20 nm) ولغرض تتفيذ البرنامج يجب مر اعاة قيم المتغير ات وات و القوات اعد الآتية :

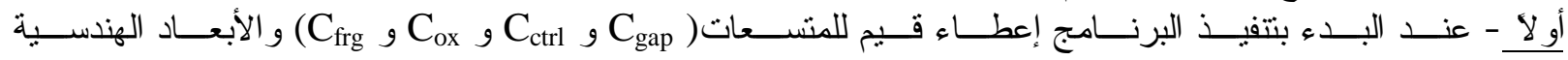
(1) و الموضحة في الثكل (1) و المذكورة قيمها في الجدول (1)

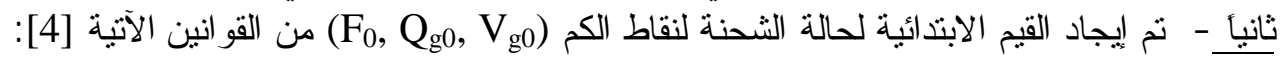

$\mathrm{V}_{\mathrm{g} 0}=\mathrm{q} /\left(\mathrm{C}_{\mathrm{ctrl}}+\mathrm{C}_{\mathrm{ox}}\right)=0.25 \quad$ Volt

$\mathrm{Q}_{\mathrm{g} 0}=\left(\sum_{\mathrm{i}=1}^{25} \mathrm{C}_{\text {ctrl }-\mathrm{i}}+\mathrm{C}_{\mathrm{frg}}\right) \mathrm{V}_{\mathrm{g} 0}=2.5825 \times 10^{-19}$ Coulomb $(\mathrm{i}=1,2, \ldots 9)$

$\mathrm{F}_{0}=-0.5 \times \mathrm{Q}_{\mathrm{g} 0} \times \mathrm{V}_{\mathrm{g} 0}=-3.228125 \times 10^{-20} \quad$ Joule 


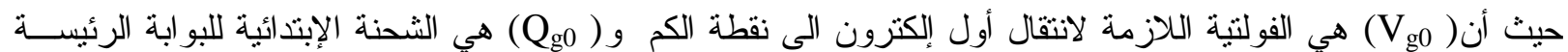
و (F0) تمثل الطاقة الكلية للنظام قبل التنفيق. ثالثئ - ولحالة انحياز جديدة : • ت تم إيجاد قيم (Vot-i

$-\mathrm{q}\left[\mathrm{n}_{\mathrm{i}}\right]=[\mathrm{C}]\left[\mathrm{V}_{\mathrm{dot}-\mathrm{i}}\right]$

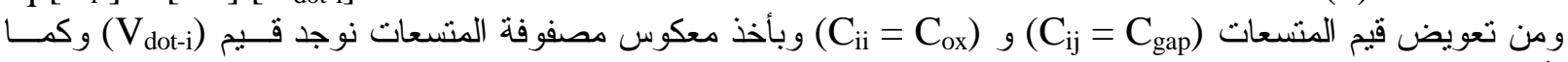
: يأني

$\left[\mathrm{V}_{\text {dot- }-1}\right]_{1 \times 25}=-\mathrm{q}[\mathrm{C}]^{-1}{ }_{25 \times 25}\left[\mathrm{n}_{\mathrm{i}}\right]_{1 \times 25}$

حيث تم الأخذ بنظر الاعتبار التنفيق بين النقاط المتجاورة كما في الثكل (1) عبر (C) (C) و التنفيق من القناة لنقطة

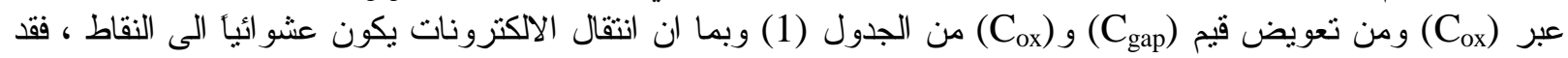

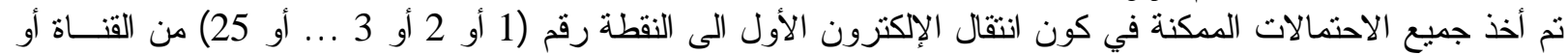
من نقاط الكم المجاورة ، ويتم تحديد أي من هذه النقاط حسب قيمة (tmin) و الذي سيتم ذكره لاحقاً ، وبعبارة أخرى (أمسا . $\left(\mathrm{n}_{25}=1 \ldots \mathrm{n}_{2}=1\right.$ أو $\mathrm{n}_{1}=1$

الجدول (1) قيم المتسعات المستخرجة لابعاد معاملات هندسية مختلفة للذاكر ات النانوبلورية [4].

\begin{tabular}{|l|l|l|l|l|l|l|}
\hline & Split 1 & Split 2 & Split 3 & Split 4 & Split 5 & Split 6 \\
\hline$T_{\text {ox }}(\mathrm{nm})$ & 2.5 & 2.5 & 2.5 & 2.5 & 2.5 & 2.5 \\
$T_{\text {ctrl }}(\mathrm{nm})$ & 20 & 20 & 20 & 20 & 20 & 10 \\
$T_{\text {qd }}(\mathrm{nm})$ & 4 & 4 & 4 & 3 & 5 & 4 \\
$T_{\text {gap }}(\mathrm{nm})$ & 4 & 2 & 6 & 4 & 4 & 4 \\
$C_{\text {ox }}(\mathrm{aF})$ & 0.655 & 0.528 & 0.744 & 0.415 & 0.952 & 0.655 \\
$C_{\text {ctrl }}(\mathrm{aF})$ & 0.160 & 0.107 & 0.213 & 0.114 & 0.214 & 0.306 \\
$C_{\text {gap }}(\mathrm{aF})$ & 0.282 & 0.516 & 0.163 & 0.179 & 0.370 & 0.261 \\
$C_{\text {frg }}(\mathrm{aF})$ & 0.631 & 0.006 & 3.213 & 1.125 & 0.244 & 1.224 \\
$C_{\text {qd-to-ch1 }}\left(\mu \mathrm{F} \mathrm{cm}^{-2}\right)$ & 2.04 & 1.96 & 2.06 & 1.83 & 2.17 & 2.04 \\
$C_{\text {qd-to-ch2 }}\left(\mu \mathrm{F} \mathrm{cm}^{-2}\right)$ & 0.825 & 1.15 & 0.654 & 0.775 & 0.860 & 0.825 \\
$C_{\text {qd-to-ch33 }}\left(\mu \mathrm{F} \mathrm{cm}^{-2}\right)$ & 0.407 & 0.728 & 0.281 & 0.398 & 0.411 & 0.405 \\
$C_{\text {gate-to-ch2 }}\left(\mu \mathrm{F} \mathrm{cm}^{-2}\right)$ & 0.119 & 0.003 & 0.343 & 0.259 & 0.039 & 0.231 \\
$C_{\text {gate-to-ch3 }}\left(\mu \mathrm{F} \mathrm{cm}^{-2}\right)$ & 0.200 & 0.006 & 0.533 & 0.390 & 0.073 & 0.387 \\
\hline
\end{tabular}

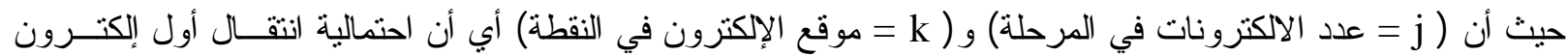

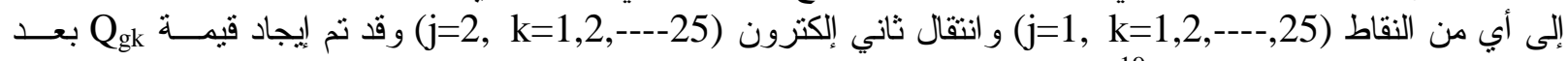
انتقال أول إلكترون ونساوي (4.1825 × $10^{-19}$ C لأية نقطة . 

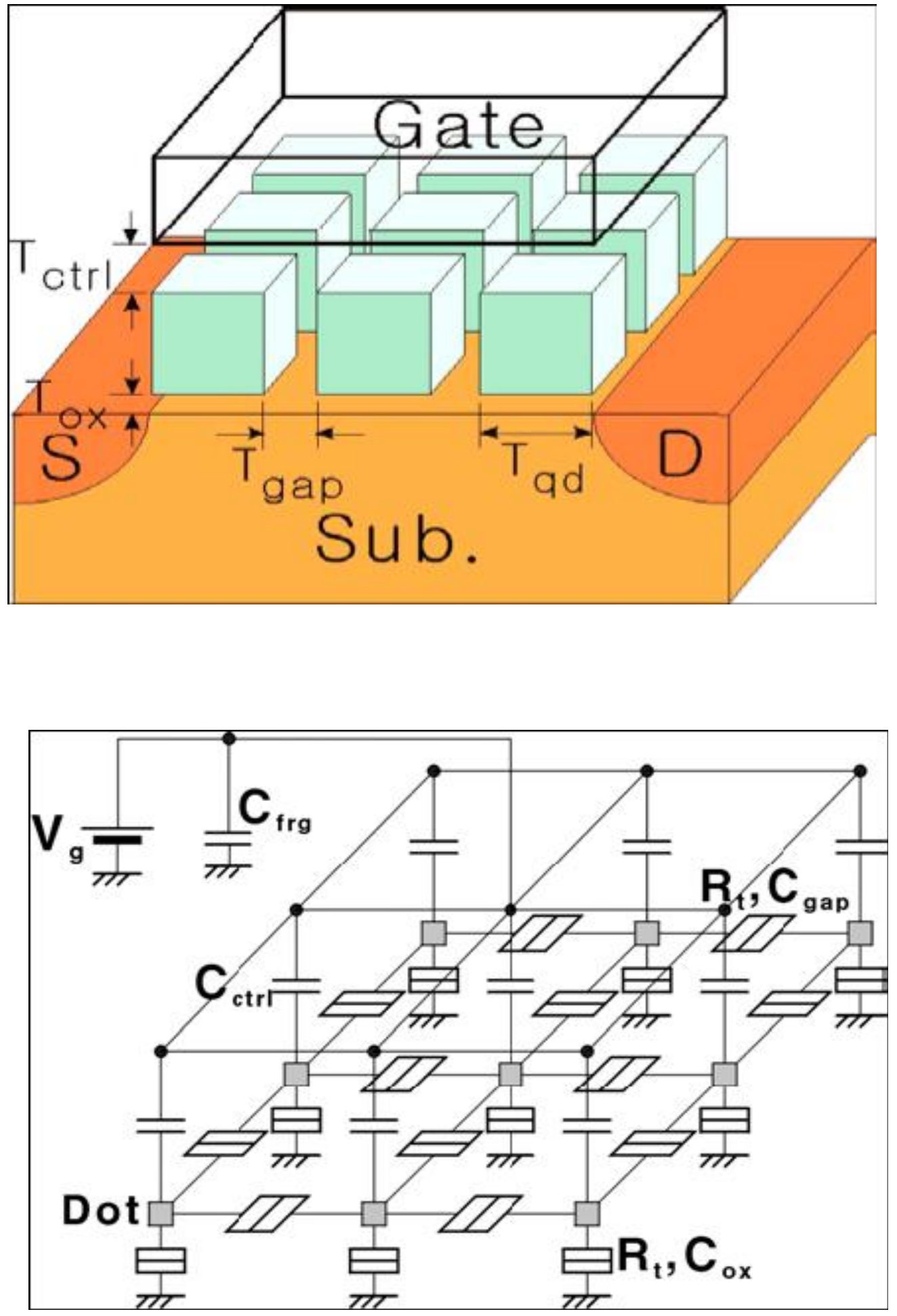

الثكل (1) : (a) رسم تخطيطي لهيكل الذاكرة النانوبلورية (b) دائرة مفارق التتفيق الاحادي (STJs)

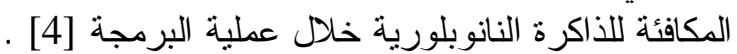


تم إيجاد قيم (

$\mathrm{V}_{\mathrm{g}-\mathrm{kj}}=\left(\mathrm{Q}_{\mathrm{kj}}+\sum \mathrm{C}_{\mathrm{ctrl}-\mathrm{i}} \times \mathrm{V}_{\text {dot-i }}\right) /\left(\sum \mathrm{C}_{\mathrm{ctrl}}+\mathrm{C}_{\mathrm{frg}}\right)$

علمأ أن فولتية النقطة تتخفض بمقدار $)\left(\frac{n k \times q}{C_{\text {ctrl }}+C_{\text {ox }}}\right)$ في حالة انتقال إلكترون اليها ويعوض هذا النقصان بالفولتية مـنـ

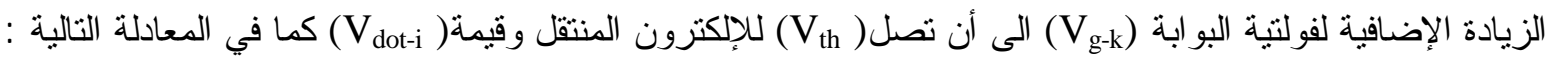

$\mathrm{V}_{\mathrm{dot}-\mathrm{i}}=\left(\mathrm{V}_{\mathrm{dot}-\mathrm{i}}-\frac{\mathrm{n} \times \mathrm{q}}{\mathrm{C}_{\mathrm{ctrl}}+\mathrm{C}_{\mathrm{ox}}}\right)$

ومن القيم السابقة نم إيجاد (Fk) لكل عمليات التنفيق الممكنة عند (STJs) عند إنتقال أول الكترون مثلا وحسب

$\mathrm{F}_{\mathrm{k}}=-0.5 \mathrm{q}\left(\sum \mathrm{n}_{\mathrm{i}} \times \mathrm{V}_{\mathrm{dot}-\mathrm{i}}\right)-0.5 \mathrm{Q}_{\mathrm{g}-\mathrm{k}} \mathrm{V}_{\mathrm{g}-\mathrm{k}}$

(Joule)

المعادلة [5]:

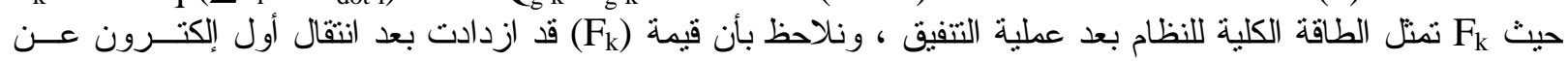

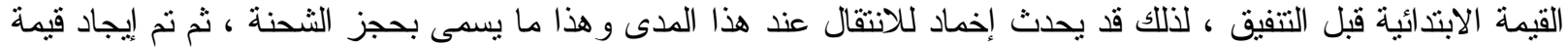

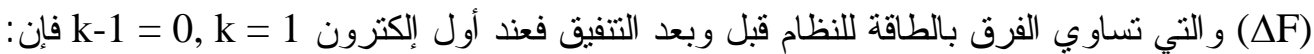

$\Delta \mathrm{F}=\mathrm{F}_{\mathrm{k}}-\mathrm{F}_{\mathrm{k}-1} \quad$ (Joule)

$\Delta \mathrm{F}=\mathrm{F}_{1}-\mathrm{F}_{0}$

وقد نم حساب قيم $)$ سابقأ.

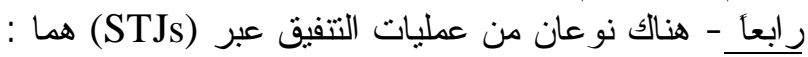

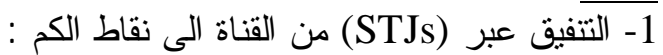

• حيث نم إيجاد قيمة المجال الكهربائي (Ex1) المسلط على أوكسيد التفيق في ( تركيب MOS (ST) ) من المعادلة :

$\operatorname{Eox}_{\text {ch-d ik }}=\left(\mathrm{V}_{\text {dot-i }}-\mathrm{V}_{\mathrm{FB}}\right) / \mathrm{T}_{\mathrm{ox}} \quad(\mathrm{V} / \mathrm{m})$

مع العلم أن (Flat Band Voltage -V هي فولتية الحزمة المستوية ، وتم إيجادها بالطريقة الاتية :

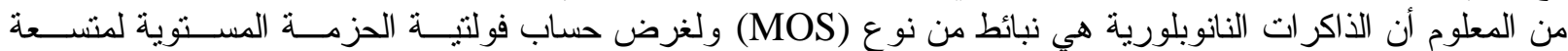
الــ(MOS) نفرض ما يأتي [34]:

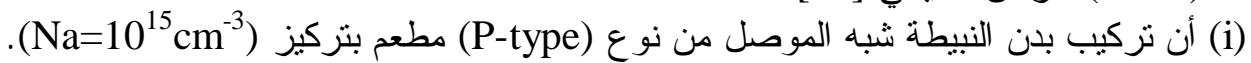
(ii) (iii) لقد تم إيجاد الفرق في دالة الثغل بين البو ابة و البدن وقيمتها (iil) $\mathrm{C}_{\text {ox }}=\frac{\varepsilon_{o x}}{\mathrm{~T}_{\mathrm{ox}}}=1.3806 \mu \mathrm{F} / \mathrm{cm}^{2}$

$\mathrm{Q}_{\mathrm{ss}}^{\prime}=\mathrm{Q}_{\mathrm{ss}} \times \mathrm{q}=2.56 \times 10^{-7} \quad$ Coulomb $/ \mathrm{cm}^{2}$ وكثافة شحنة سطح الأوكسيد المكافئة من : ومن ثم إيجاد فولتية الحزمة المستوية من : $\mathrm{V}_{\mathrm{FB}}=\phi_{m s}-\frac{\mathrm{Q}_{\mathrm{ss}}^{\prime}}{\mathrm{C}_{\mathrm{ox}}}=-1.21542626 \quad$ Volts

وبعد إيجاد قيم المجال الكهربائي

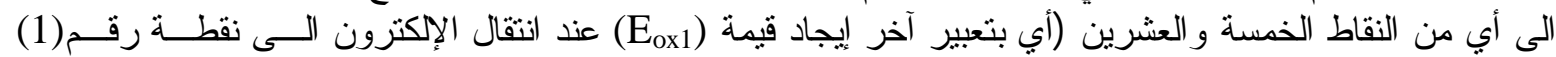

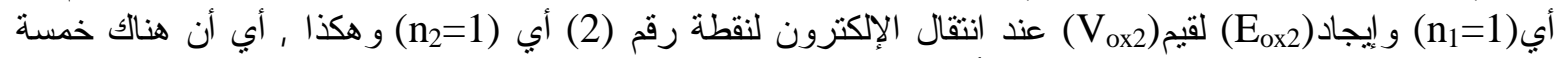

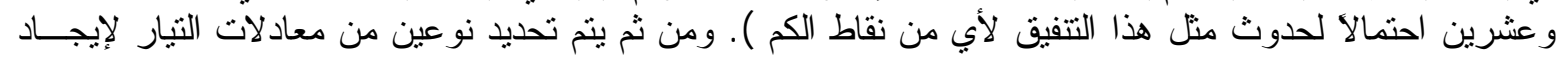

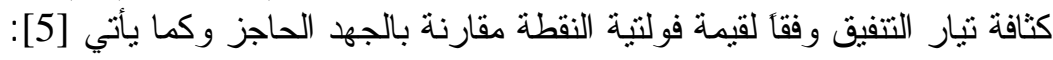
(a) معادلة تيار التنفيق المباشر 
تم استخدام المعادلة التالية في حالة كون جهد النقطة أقل من الجهد الحاجز .

$$
\begin{aligned}
& \mathrm{V}_{\mathrm{ox}}<\phi_{b} \\
& \mathrm{~J}_{\text {tunnel,direct }}=\mathrm{A} \frac{\mathrm{V}_{\mathrm{ox}}}{\phi_{b}}\left(\frac{2 \phi_{b}}{\mathrm{~V}_{\mathrm{ox}}}-1\right) \mathrm{E}_{\mathrm{ox}}^{2} \times \exp \left(\frac{-\mathrm{B}\left[1-\left(1-\mathrm{V}_{\mathrm{ox}} / \phi_{b}\right)^{1.5}\right]}{\mathrm{E}_{\mathrm{ox}}}\right) \\
& \text { (Fowler - Nordheim F/N) معادلة فولر - نوردهام (b) } \\
& \mathrm{J}_{\text {tunnel, } \mathrm{F} / \mathrm{N}}=\mathrm{A} \mathrm{E}_{\mathrm{ox}}^{2} \exp \left(\frac{-\mathrm{B}}{\mathrm{E}_{\mathrm{ox}}}\right) \\
& \text { تم استخدام المعادلة التالية في حالة كون جهد النقطة أكبر من الجهد الحاجز [5]. } \\
& \mathrm{A}=\mathrm{q}^{3} /\left(8 \pi \mathrm{h}\left(\mathrm{q} \phi_{b}\right)\right)=4.9559 \times 10^{-7} \\
& \mathrm{~A} / \mathrm{cm}^{2} \\
& \mathrm{~B}=8 \pi\left(2 \mathrm{~m}_{\mathrm{ox}}\right)^{1 / 2}\left(\mathrm{q} \phi_{b}\right)^{3 / 2} /(3 \mathrm{hq})=241.3272 \quad \mathrm{MV} / \mathrm{cm}
\end{aligned}
$$

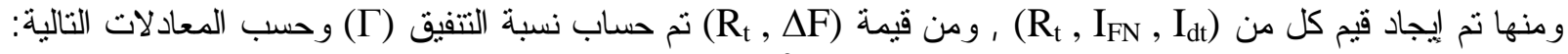

$$
\begin{aligned}
& \mathrm{I}=\mathrm{J} \times \text { area } \\
& \text { area }=\left(\mathrm{T}_{\mathrm{qd}} \times \mathrm{T}_{\mathrm{qd}}\right) \mathrm{cm}^{2} \\
& \mathrm{R}_{\mathrm{t}}=\mathrm{V}_{\text {dot }} / \mathrm{I} \\
& \Gamma=-\Delta \mathrm{F} / \mathrm{q}^{2} \mathrm{R}_{\mathrm{t}}\left[1-\exp \left(\Delta \mathrm{F} / \mathrm{K}_{\mathrm{B}} \mathrm{T}\right)\right]
\end{aligned}
$$

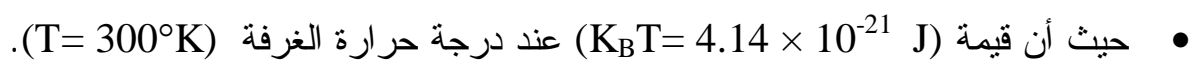
• بعد حساب قيم نسب التتفيق (C) لكل عمليات التتفيق عبر (STJs) بين القناة والنقطة ولكل احتمالات انتقال

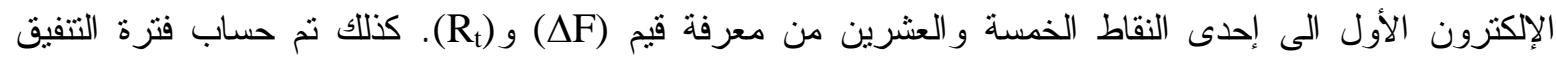
(tunnel interval) كثافة الاحتمالية له[6]: الكل

$$
\mathrm{F}(\mathrm{t})=\Gamma \exp (-\Gamma \mathrm{t})
$$

ومن ثم تحسب فترة التنفيق (tandom number generator = r) باستخدام مولد الرقم العشو ائي (rand ) بمدى > 0)

$$
\text { : [6] } \mathrm{r} \leq 1)
$$

$\mathrm{t}_{\text {tun }}=\frac{-1}{\Gamma^{ \pm}} \ln (\mathrm{r})$

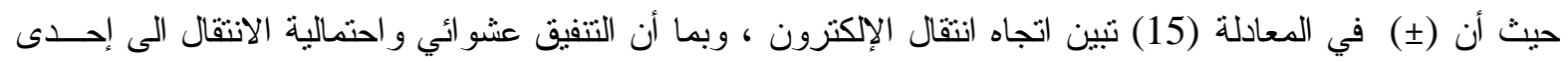
النقاط مساو, للآخر وهي [7]:

$$
\mathrm{P}(\mathrm{r})=\frac{1}{9}=0.1111
$$

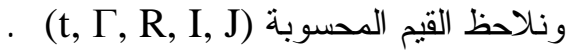

$$
\begin{aligned}
& \text { 2. احتمالية التنفيق بين نقاط الكم : } \\
& \text { تم إيجاد قيمة المجال الكهربائي (Ex) عبر الأوكسيد بين النقطنين من المعادلة [6]: } \\
& \operatorname{Eox}_{\mathrm{d}-\mathrm{d}}=\Delta \mathrm{V}_{\mathrm{dot}} / \mathrm{T}_{\mathrm{gap}} \\
& \text { ومن المعلوم أن }\left(\Delta V_{\text {dot }}\right) \text { هي الفرق بين جهد نقطتين متجاورتين و هنالك نوعان من تبار ات التتفيق : }
\end{aligned}
$$

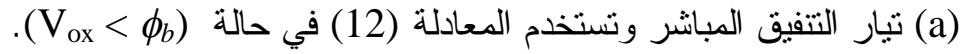

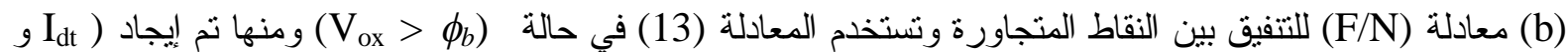
(Rt و من القو انين الاتية

$$
\begin{array}{ll}
\mathrm{I}=\mathrm{J} \times \text { area } & \text { area }=\mathrm{T}_{\mathrm{qd}} \times \mathrm{T}_{\mathrm{qd}} \\
\mathrm{R}_{\mathrm{t}}=\Delta \mathrm{V}_{\text {dot }} / \mathrm{I} &
\end{array}
$$

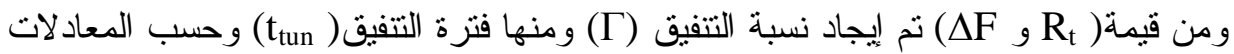$$
\Gamma=-\Delta \mathrm{F} / \mathrm{q}^{2} \mathrm{R}_{\mathrm{t}}\left[1-\exp \left(\Delta \mathrm{F} / \mathrm{K}_{\mathrm{B}} \mathrm{T}\right)\right]
$$ 
$\mathrm{t}_{\mathrm{tun}}=-\frac{1}{\Gamma} \ln (\mathrm{r})$

وقد تم أيجاد جميع القيم المحسوبة ، وكان عدد احتمالات التتفيق (80 حالة) بين النقاط.

• بعد أن تم الحصول على مجموعة احتمالات لفترة التنفيق (ttun) لانتقال أول الكترون من كافة الاحتمالات الممكنة

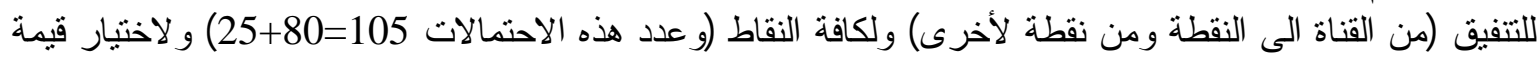

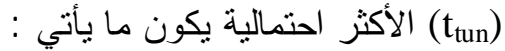

1- حسب نظرية (torthodox) ولغرض الاكن حصول تنفيق يجب أن يتحقق الشرط التالي [6]:

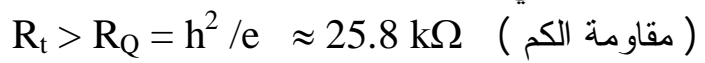

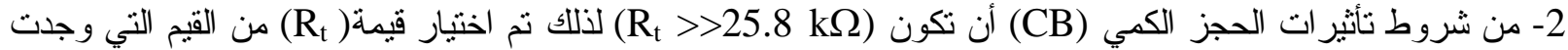

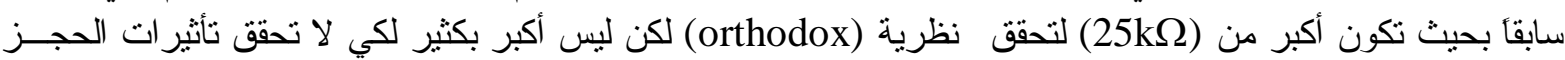

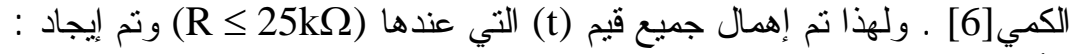

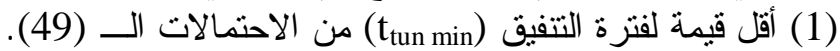

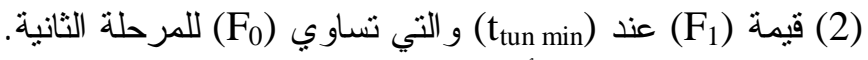

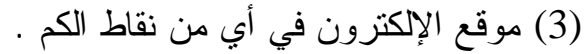

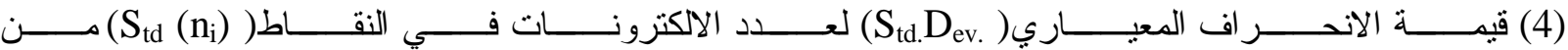
المعادلة [6]:

$\mathrm{S}_{\mathrm{td}}=\left(\frac{1}{\mathrm{n}-1} \sum\left(\mathrm{x}_{\mathrm{i}}-\mathrm{x}\right)^{2}\right)^{1 / 2}$

$V_{\text {th-k }}=m\left(\frac{n \times q}{25 C_{\text {ctrl }}+C_{\text {frg }} \frac{C_{o x}+C_{c t r l}}{C_{o x}}}\right)$

(5) و لإيجاد قيمة (Vth) للالكترون المنتقل وحسب المعادلة النالية :

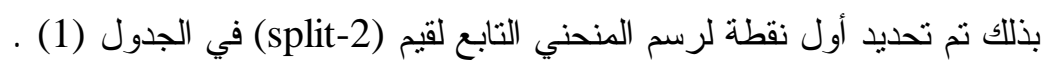

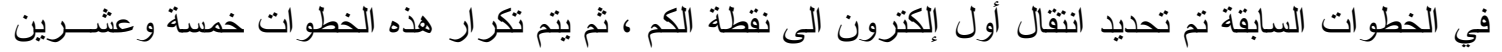

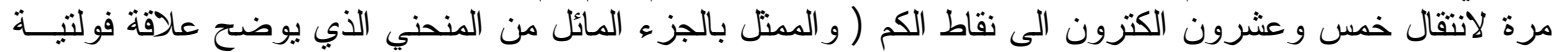

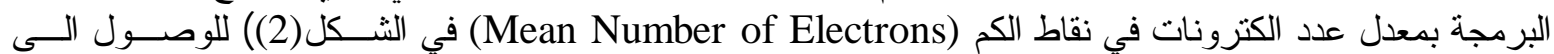

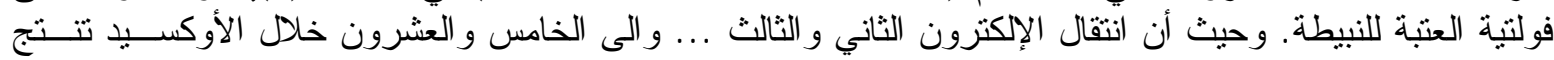
$\mathrm{Q}_{\mathrm{g}-\mathrm{k}}=\mathrm{Q}_{\mathrm{g} 0}+\mathrm{jq}$

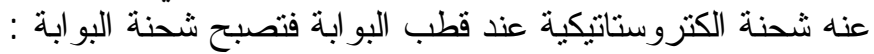

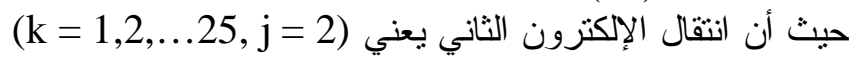

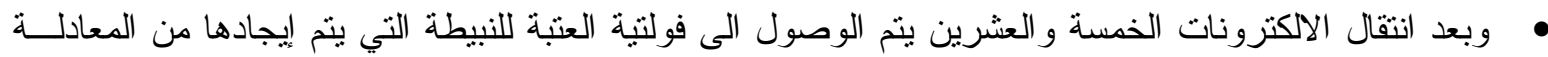

$\mathrm{V}_{\text {th }}=\mathrm{m}\left(\frac{25 \times \mathrm{q}}{25 \mathrm{C}_{\text {ctrl }}+\mathrm{C}_{\text {frg }}}\right) \quad \mathrm{m}=1,2, \ldots$

النالية [6]:

• بعد الوصول الى فولتية العتبة للنبيطة ، ولكي ينتقل أول إلكترون للمرحلة الثانية (أي الإلكتــرون رقــم (26))

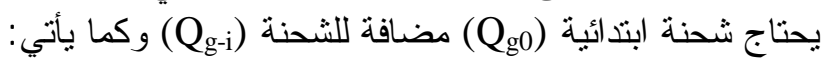

$\mathrm{Q}_{\mathrm{g}-\mathrm{m}}=\mathrm{mQ}_{\mathrm{g} 0}+\mathrm{jq}$

وبتكرار جميع الخطوات السابقة للمرحلة الأولى أي انتقال خمس وعشرون الكترون أخرى للوصول الى فولتية العتبة

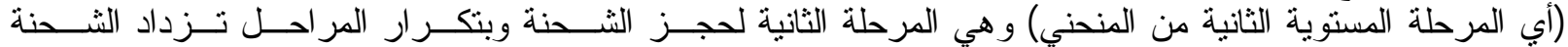

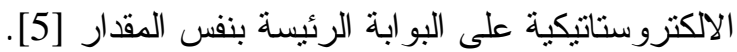


ومنها تحسب قيمة (V) وبتكر ار هذه العمليات الى أن تصل فولنية (

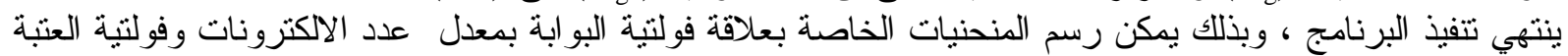

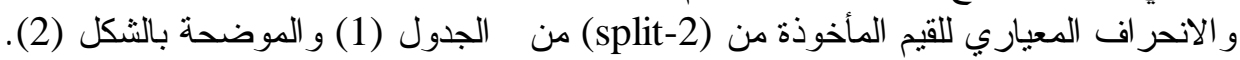

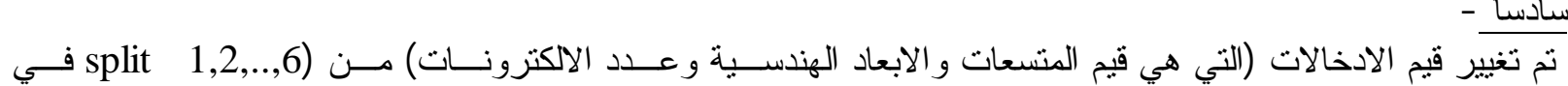

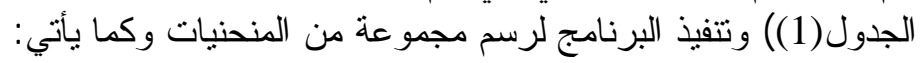

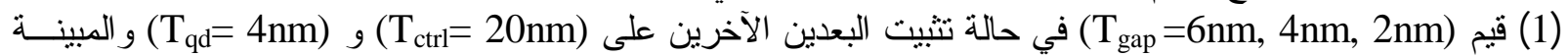

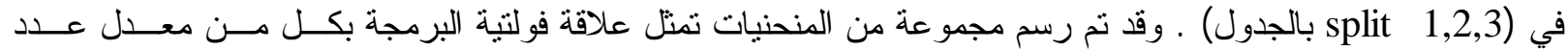

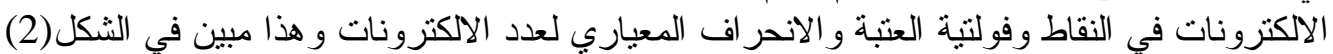

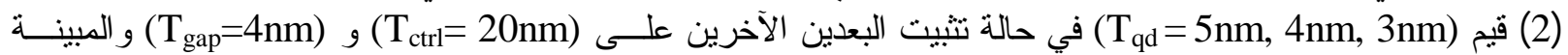

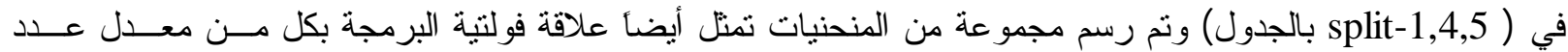

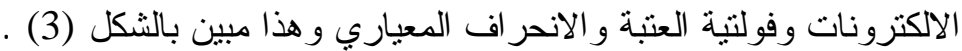

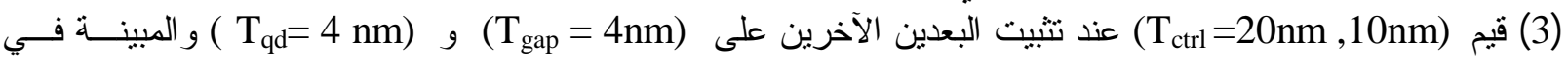

(4) split-1,6) بالجدول) وقد نم رسم مجموعة المنحنيات المذكورة سابقأ للقيم الجديدة وهذا موضح بالشكل (4).

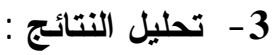

ويشمل التحليل الخو اص الاتية :

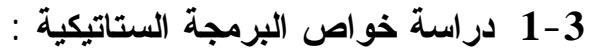

تمت در اسة تأثثر المعاملات الهندسية

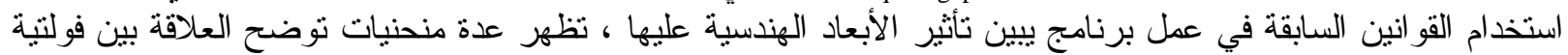

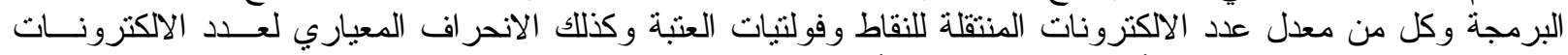

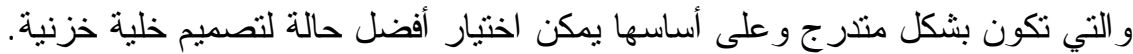
فيما يأتي تحليل تأثنير ات تغير أحد هذه المعاملات مع تثنيبت قيم المعاملات الأخرى وكما يأني:

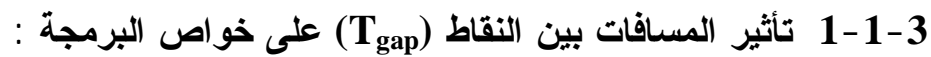

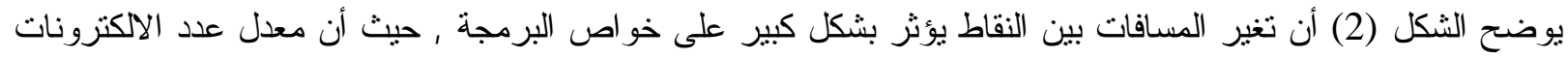

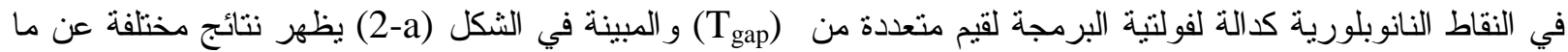

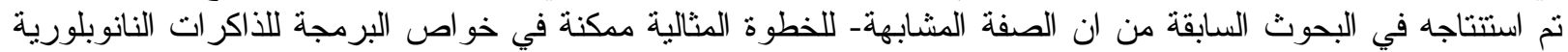

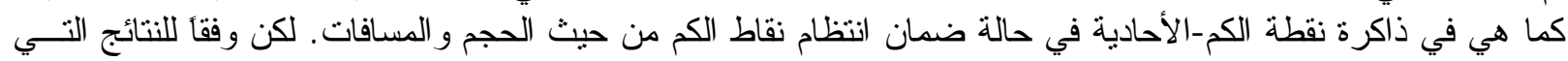

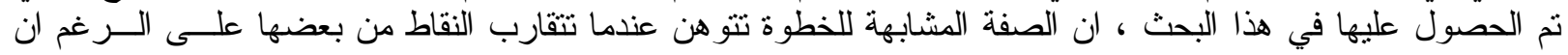

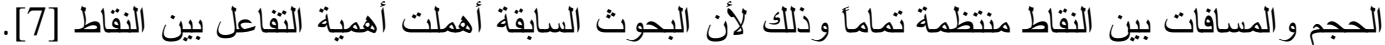

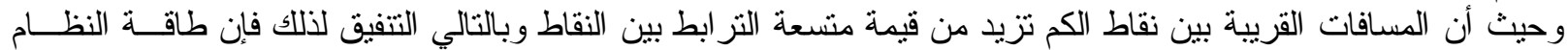

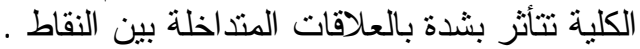

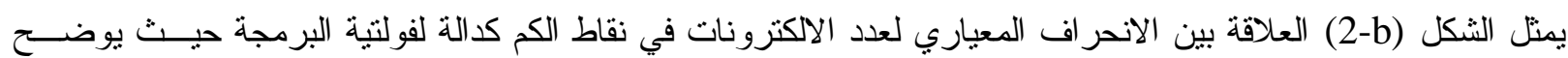

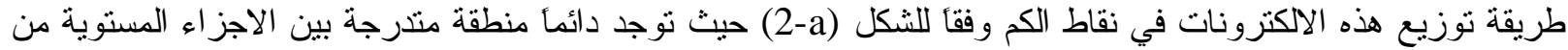

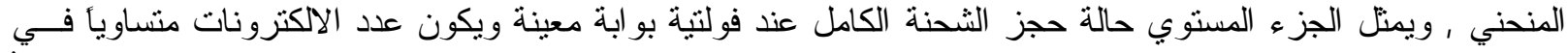

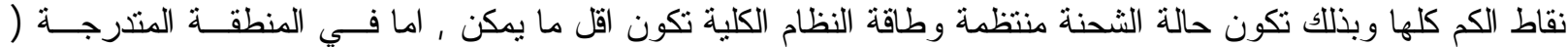

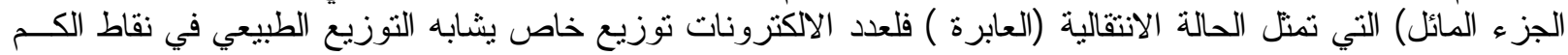

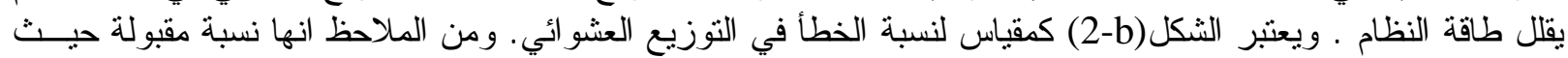

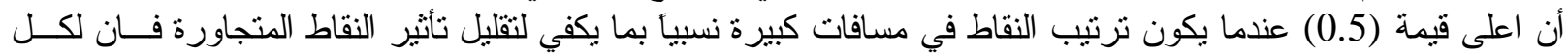

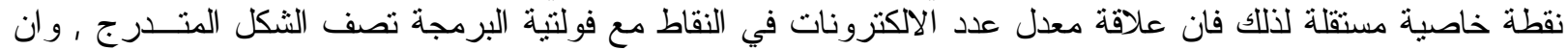

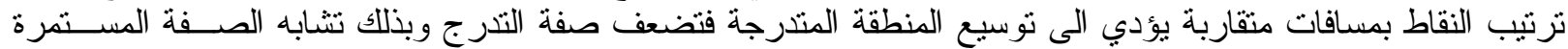

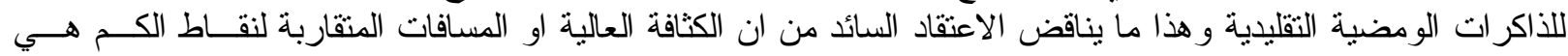




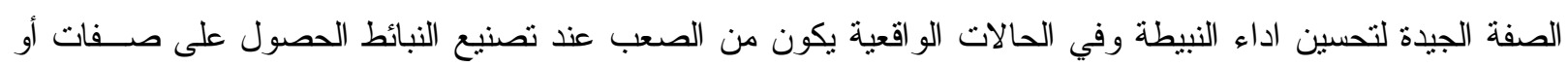

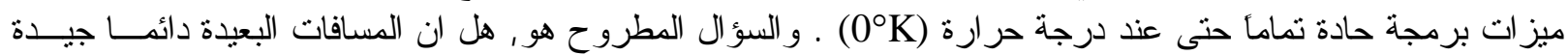

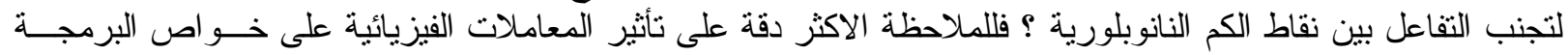

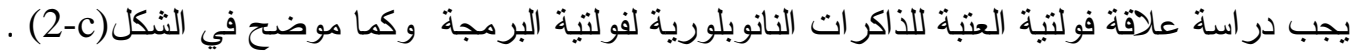
أما من جهة إمكانية تطبيق ذاكرة-الإكترون الأحادي كخلايا متعددة المستويات فمن المستحسن النية أن تكون الصفة المتدرجة

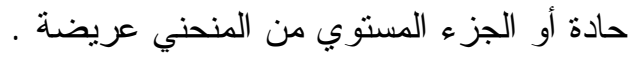

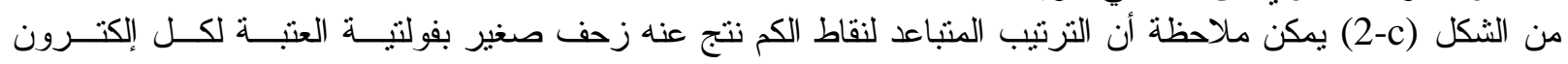
و التي تعتبر من المساوىء في (L) على منطقة القناة.

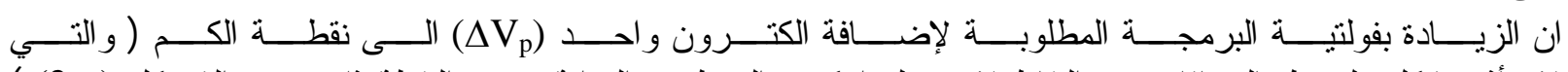

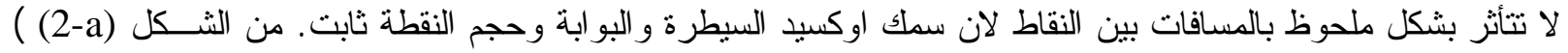

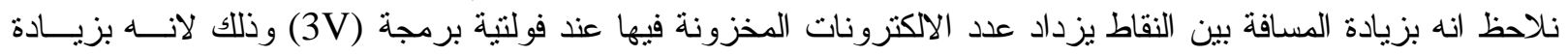

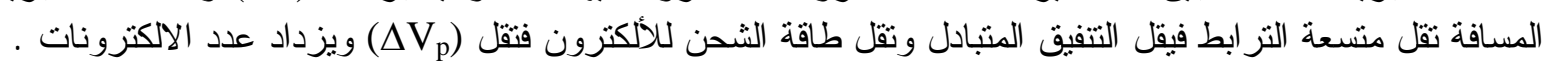

\section{: 2-1-3 على خواص البرمجة (Td}

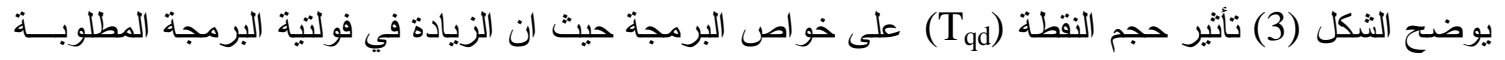

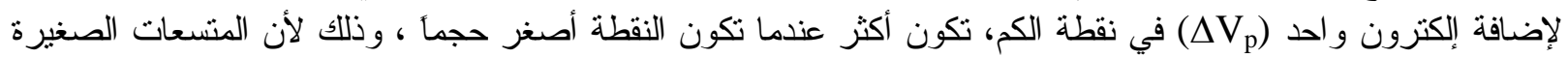

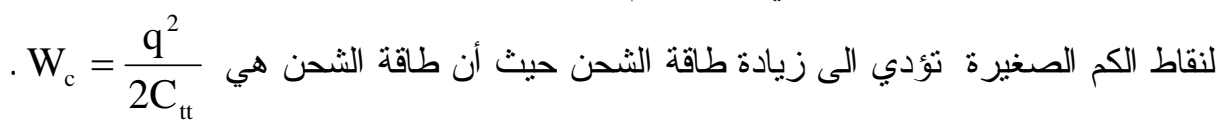

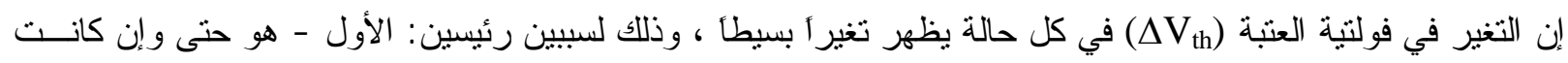

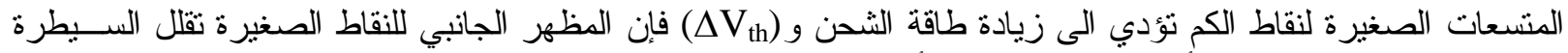

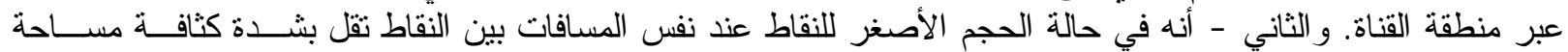

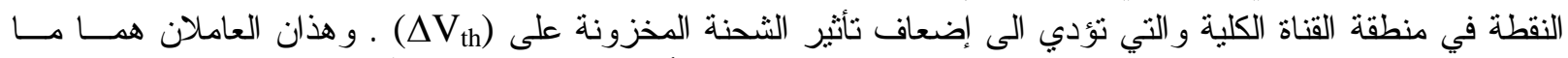

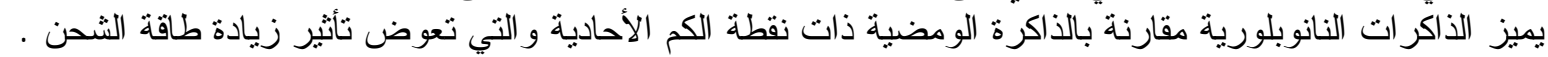

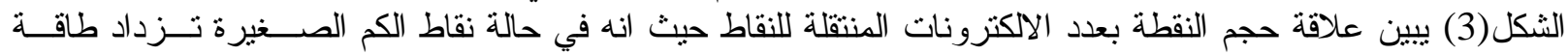

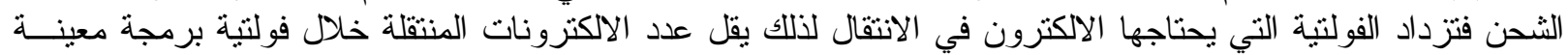
ويقل عدد مستويات الخزن .

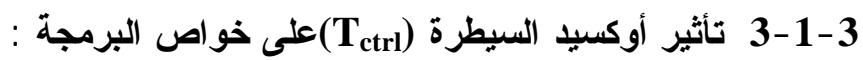

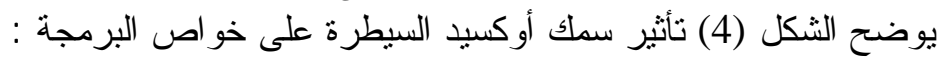
من المعروف أن زيادة

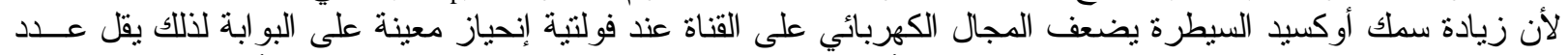

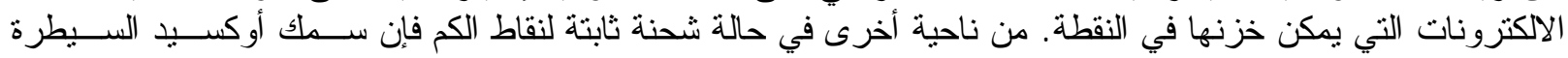

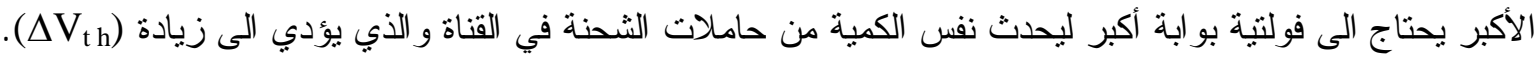

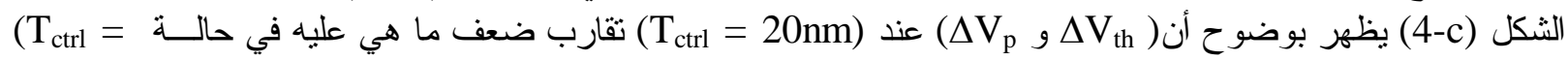

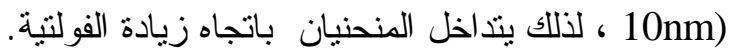

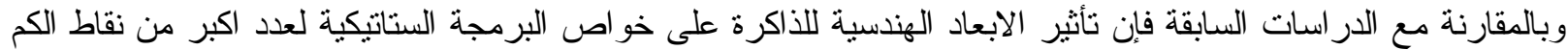

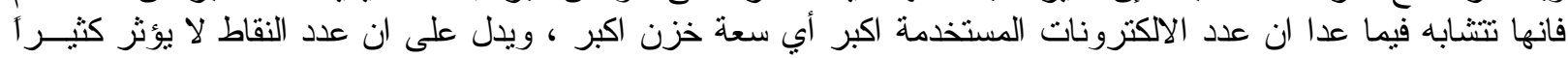

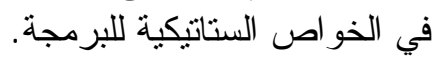

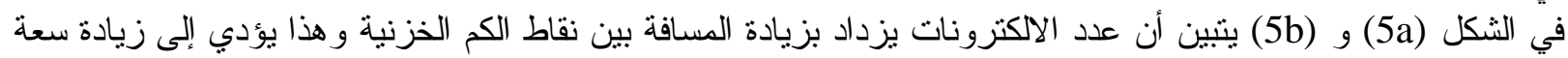

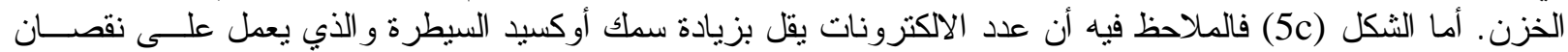




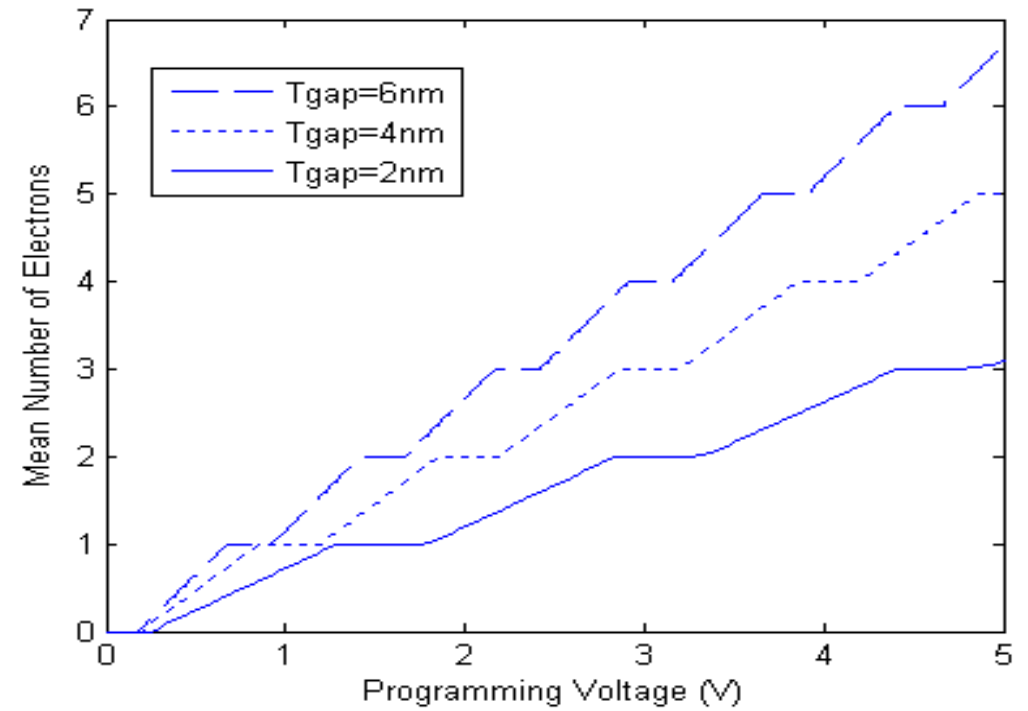

(a)

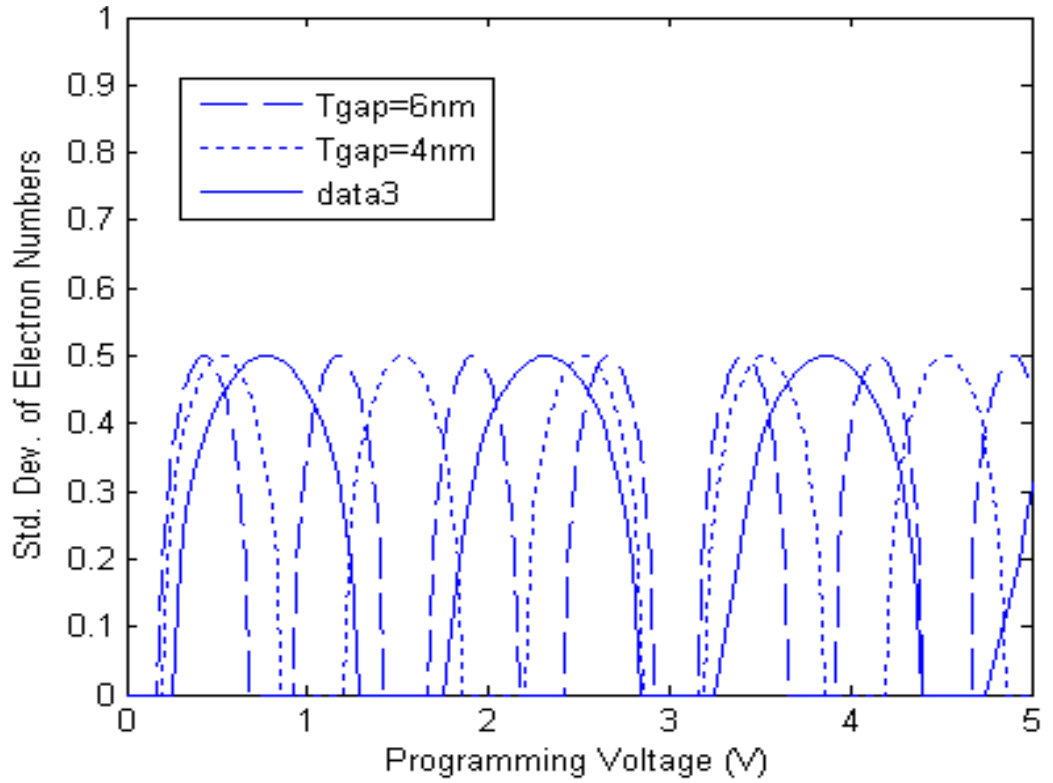

(b)

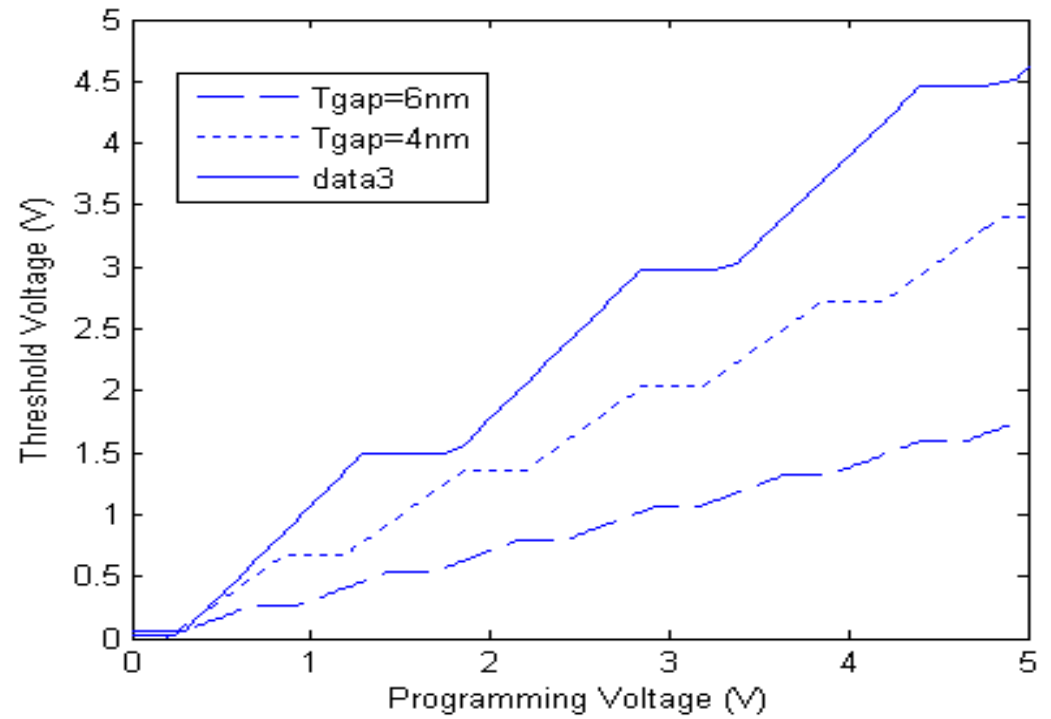

(c)

الثكل (2) علاقة المسافة بين النقاط بفولتية البرمجة الستانيكية للنموذج (5×5) 


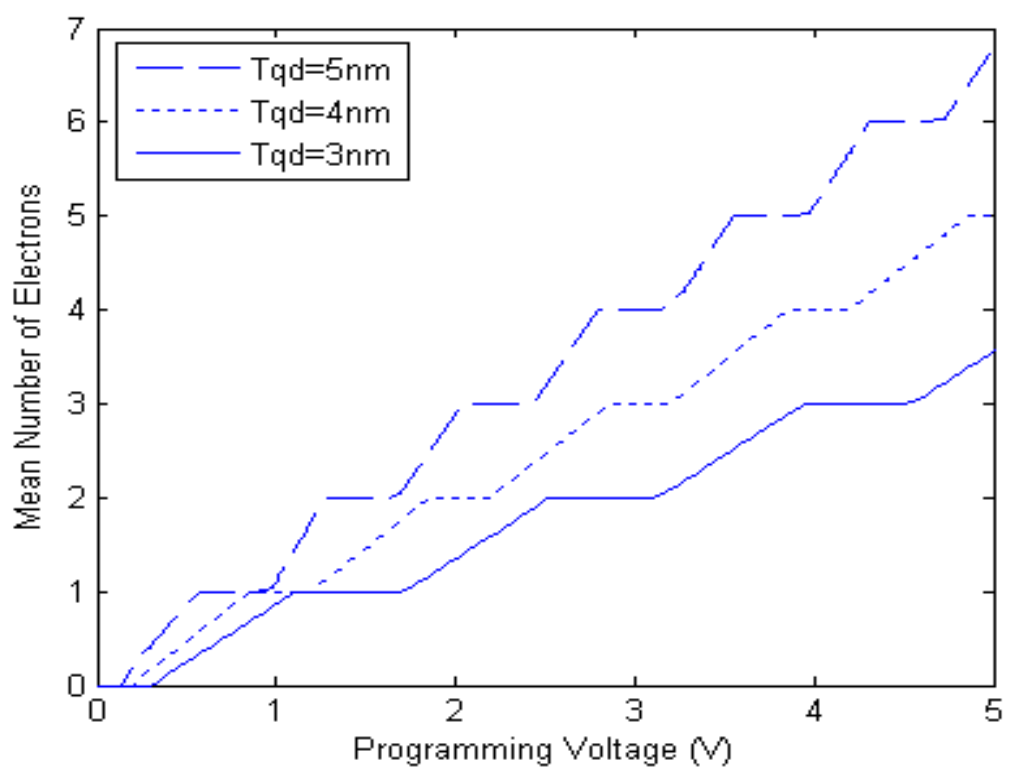

(a)

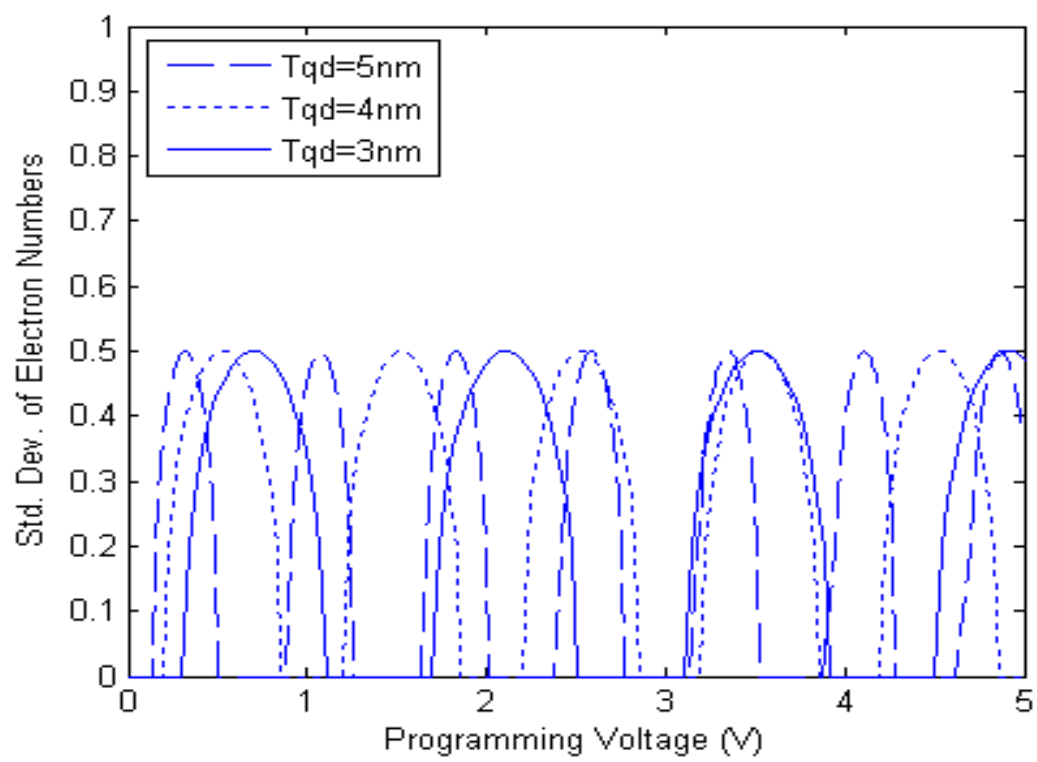

(b)

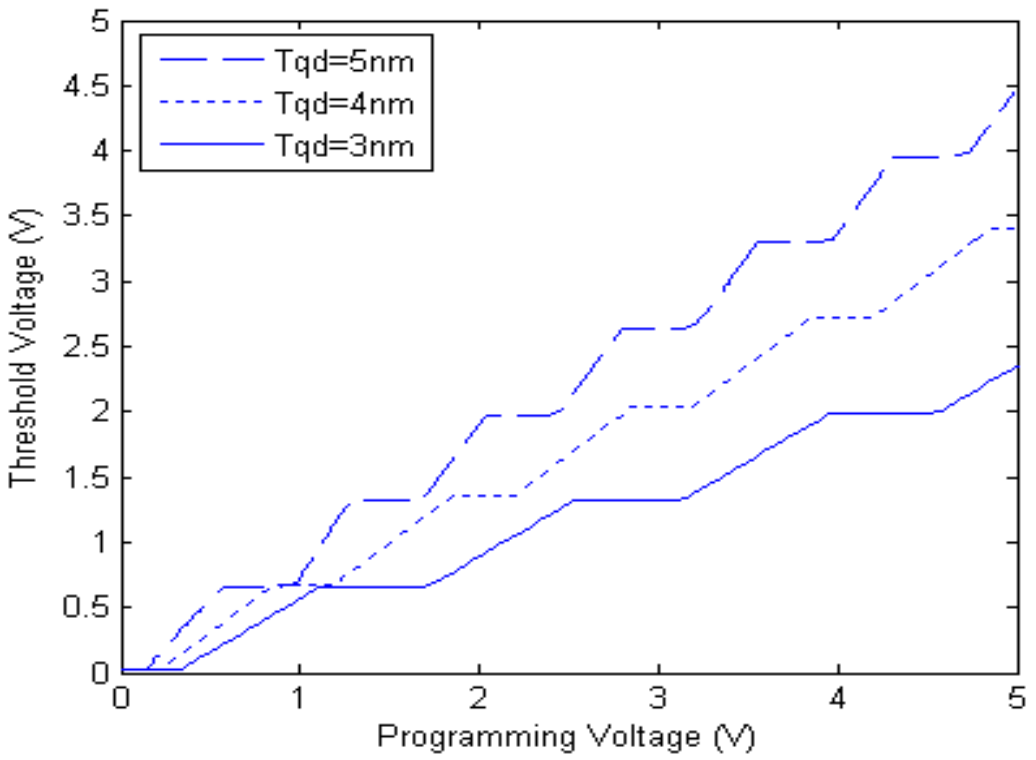

(c)

الثكل (3) علاقة حجم النقطة بخو اص البرمجة الستاتيكية للنموذج (5×5) 


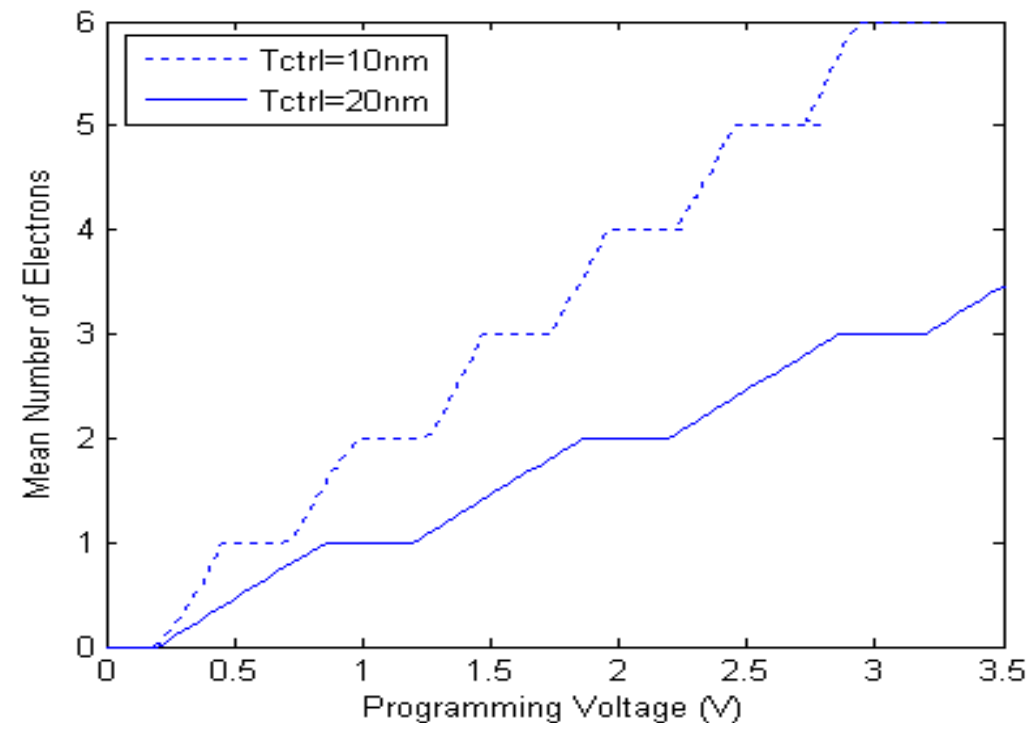

(a)

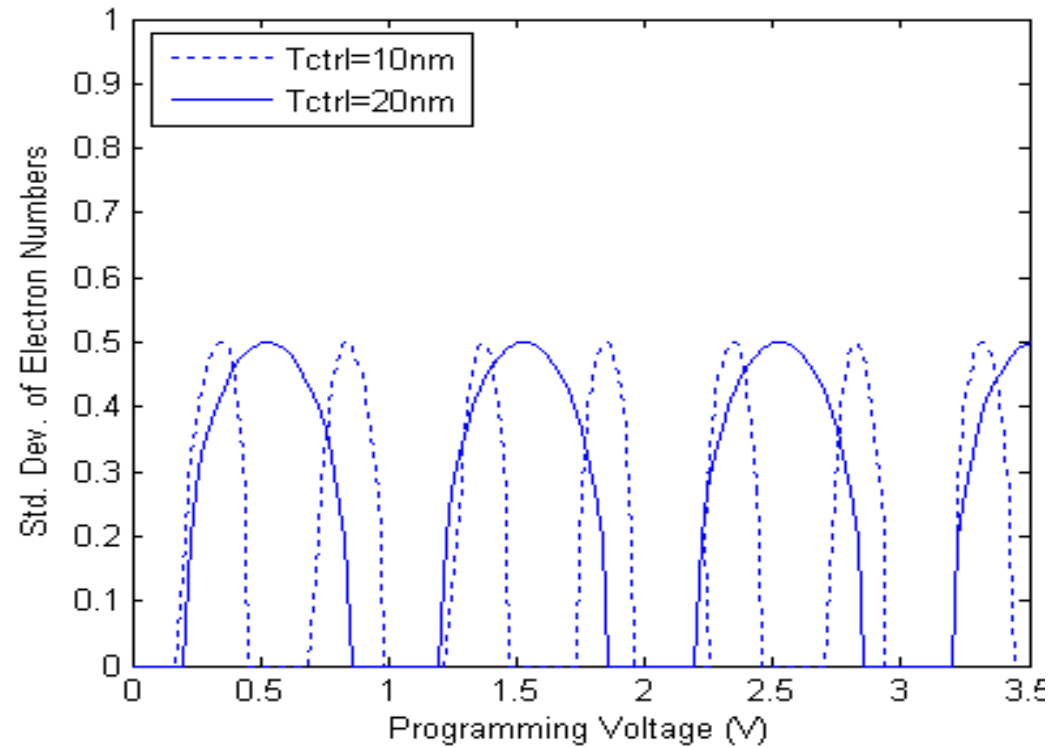

(b)

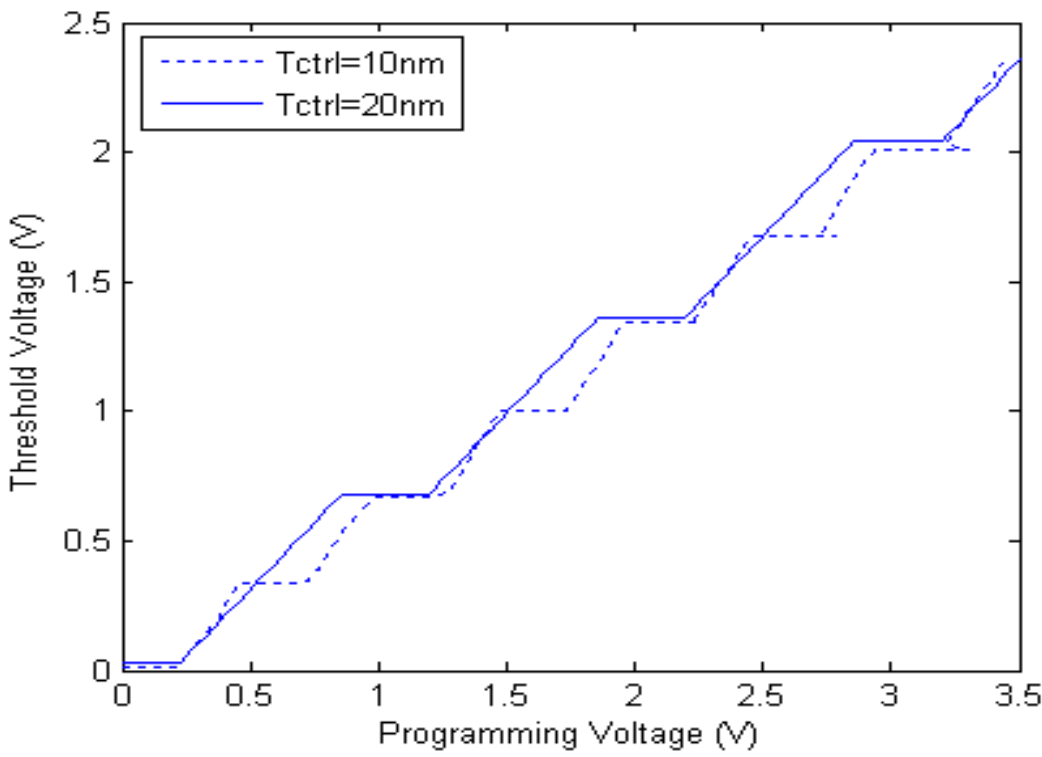

(c)

الثكل (4) علاقة سمك (وكسيد السيطرة بخواص البرمجة الستاتيكية للنموذج (5×5) 
علي : نمذجة خلايا الخزن النانو بلورية

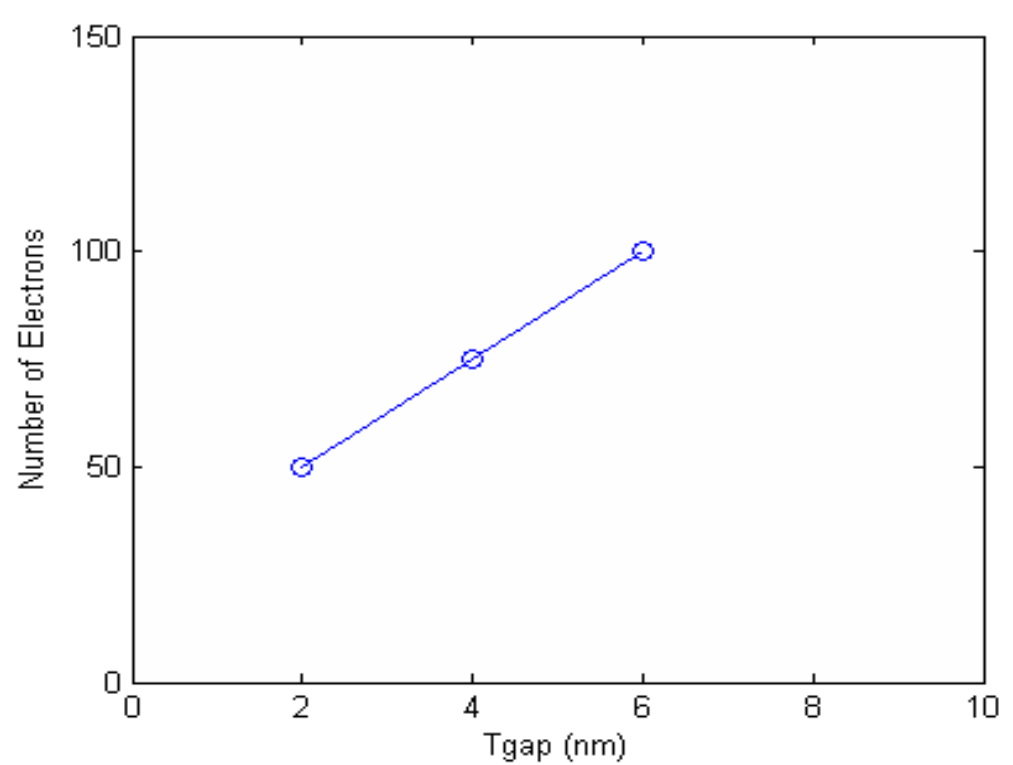

(a)

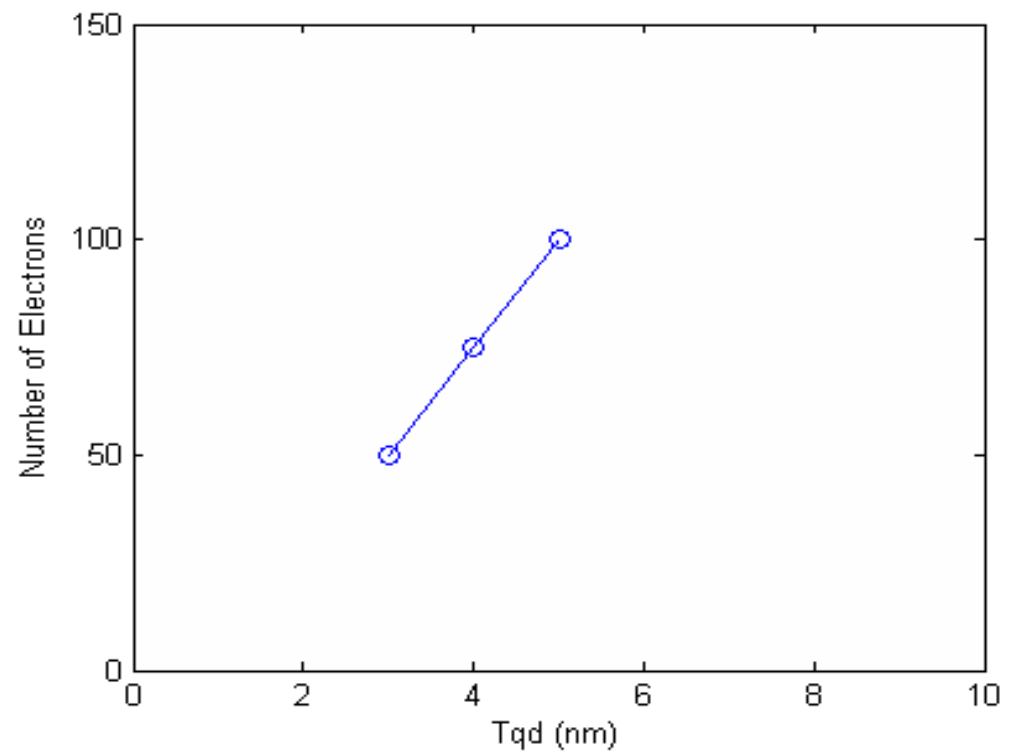

(b)

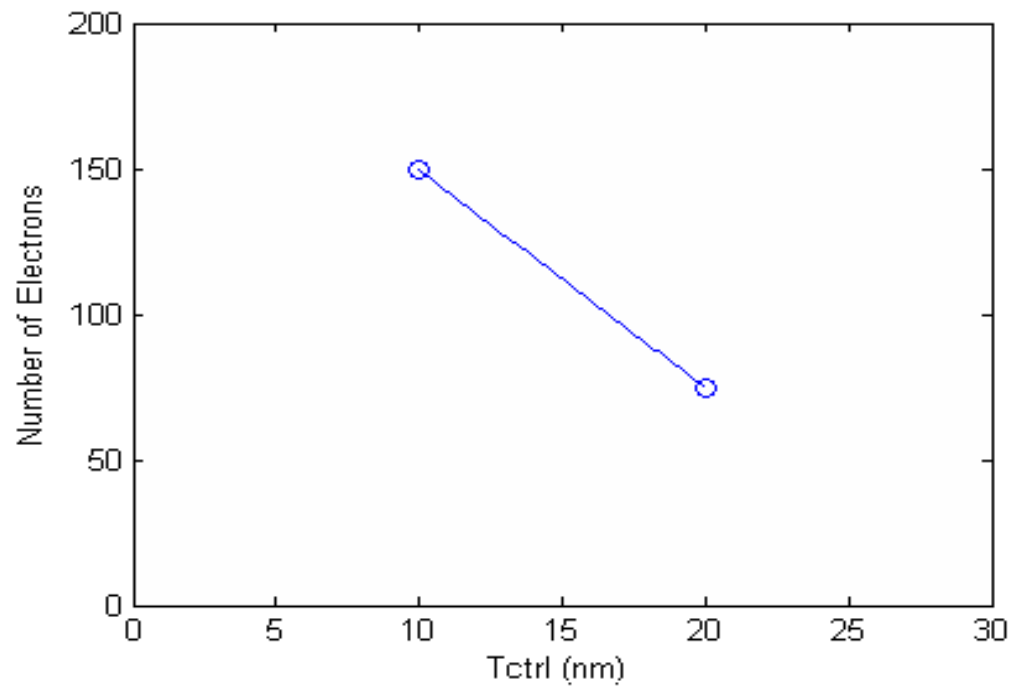

(c)

الثكل (5) العلاقة بين عدد الالكترونات في نقاط الكم و الابعاد الهنسية عند

$$
\text { للنموذج (Vg=3 V) }
$$




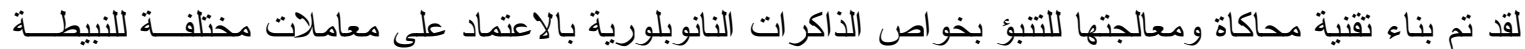

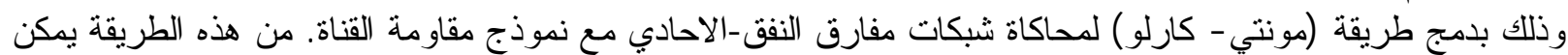

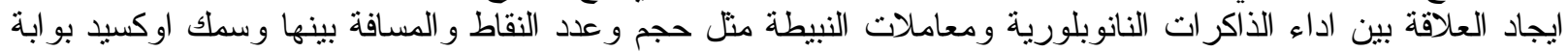

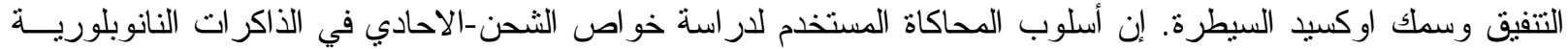

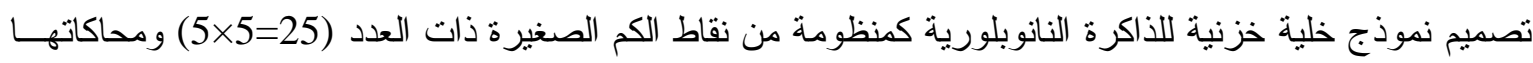

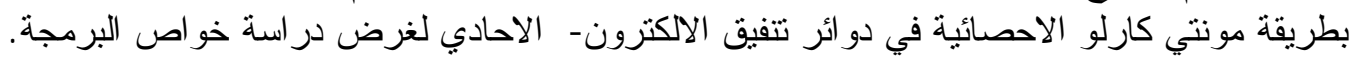

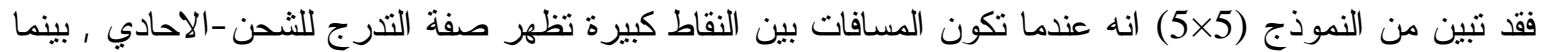

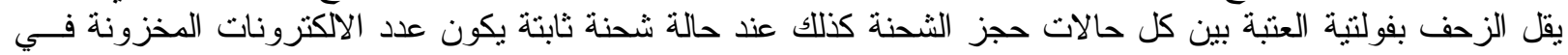

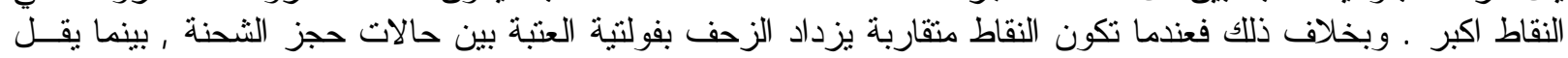

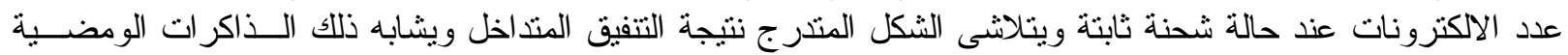

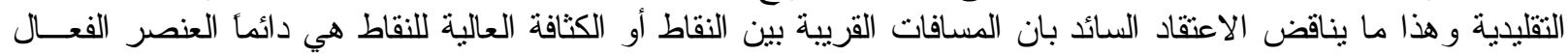

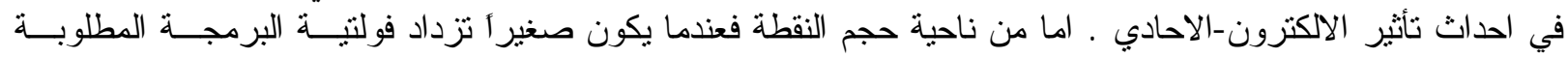

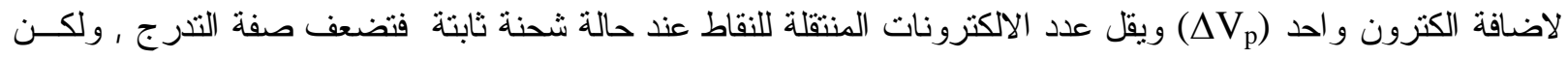

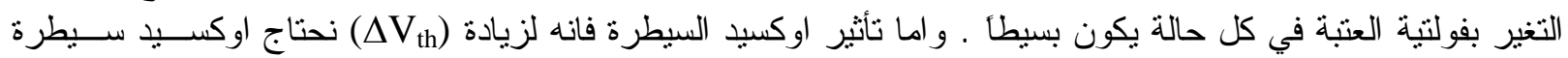

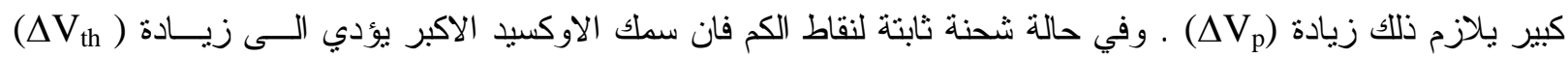

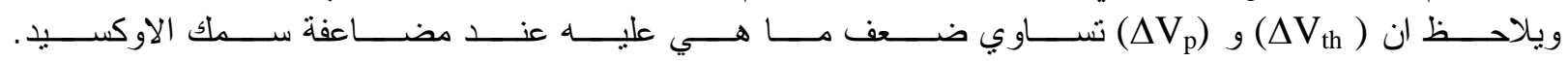

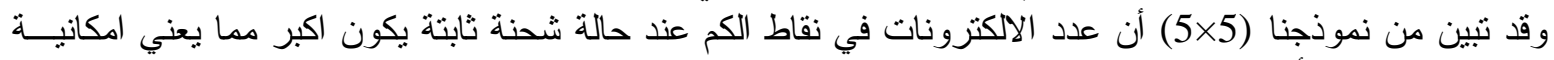
زيادة عدد بتات الخزن أي زيادة الكثافة الخزنية .

: المصادر

1. Yu Y.S., Choi B.H., Oh J.H., Hwang S.W. \& Ahn D., December 2001,"Single Electron Memory with Silicon Self - Asseubled", Journal of the Korean Physical Society, Vol. 39, PP. 527 N S29.

2. $\quad$ Sung S.K., Kim D.H., Sim J.S., kim K.R., Lee Y.K., Lee J.D., Chae S. D., Kim B.M. \& Park B.G., April 2002, "Single - Electron MOS Memory with a Defined Quantum Dot Based on Conventional VLSI Technology", The Japan Society of Applied Physics, Vol.41, PP. 2606 - 2610.

3. Brault J., Saitoh M., Kim I., Yanagidaira K. \& Hiramoto T.,2002, "Fabrication of nano scale MOSFETs for the Realization of Single-Electron Memories", Limms/ Cnrs - IIS, University of Tokyo, 4-6-1, Komaba, Megnro, Ku, 153-8505 Tokyo Japan.

4. Sim J.S., Lee J.D. \& Park B.G., 2004, "The Simulation of Single-Charging Effects in the Programming Characteristics of Nanocrystal Memories", IOP Publishing Ltd, Seol National University.

5. Sim J.S., Kong J., Lee J.D. \& Park B.G., 2004, "Monte-Carlo Simulation of SingleElectron Nanocrystal Memories", Japanese Journal of Applied Physics, Vol.43, No.4B, PP. 2041-2045.

6. Sim J. S., Kong J., Lee J. D. \& Park B.G., 2003, "Monte-Carlo Simulation of SingleElectron Nanocrystal Memories", Extended Abstracts of the 2003 International Conference on Solid State Devices \& Materials, Tokyo, PP. 850-851.

7. Neamen D. A., 1992, "Semiconductor Physics and Devices", Richard D. Irwin, INC. Australia.

$$
\text { تم اجراء البحث في كلية الهندسة - جامعة الموصل }
$$

